\title{
The role of short term population movement in sustaining STI prevalence in remote Australian Indigenous communities
}

\author{
Ben B. Hui ${ }^{a}$, Richard T. Gray ${ }^{a}$, David P. Wilson ${ }^{a}$, James S. Ward ${ }^{b}$, Anthony M. A. Smith \\ David J. Philp ${ }^{a}$, Matthew G. Law ${ }^{a}$, Jane S. Hocking ${ }^{\text {, }}$, David G. Regan ${ }^{\mathrm{a}}$ \\ ${ }^{a}$ The Kirby Institute, University of New South Wales, Sydney, NSW 2052, Australia \\ ${ }^{b}$ Baker IDI Heart and Diabetes Institute, Alice Springs, NT 0871, Australia \\ ${ }^{c}$ Australian Research Centre in Sex, Health and Society, La Trobe University, Melbourne, Victoria 3000, \\ Australia \\ ${ }^{d}$ Centre for Women's Health, Gender and Society, The University of Melbourne, Carlton, Victoria 3053, \\ Australia \\ Email:b.hui@unsw.edu.au
}

\begin{abstract}
For almost two decades, diagnosis rates of sexually transmitted infection (STI) such as chlamydia and gonorrhoea have been notably higher for residents of remote Indigenous communities than for non-Indigenous Australians. High levels of population movement between remote communities may be a contributing factor in sustaining these high rates.
\end{abstract}

We developed an individual-based computer simulation model to study the relationship between population movement and the persistence of STIs within multiple small communities. We examine the distribution of infection across locations and the impact of mobility and periodicity on prevalence over a range of locationand time-specific screening interventions.

Our findings suggest that: 1) short-term population movement, along with periodic variations in travel patterns allow STIs to persist at a high level in multiple small populations; 2) infection is not evenly distributed across populations, with more than $80 \%$ of infection concentrated at the most populous location, despite this locations only comprising $72 \%$ of the total population at a given time; 3 ) maintaining screening coverage at the current level (44\% of population screened annually) in small locations alone has limited impact on STI prevalence in the overall population; reduction in prevalence is unlikely to be achieved unless screening coverage and frequency are increased substantially or screening is expanded to include larger regional centres prevalence is higher; 4) population mobility can influence the outcomes of location- and time-specific screening interventions; interventions should be scheduled to occur during periods of low mobility when individuals are more likely to be at home and have access to local health services.

Keywords: Mobility, STI transmission, prevalence, screening, Indigenous populations, remote communities, chlamydia, gonorrhoea 
Hui et al., The role of short term population movement in sustaining STI prevalence in remote Australian Indigenous communities

\section{INTRODUCTION}

Remote areas of Australia have a substantially higher reported rate of sexually transmissible infections (STI) than urban and regional centres. The difference is especially noticeable for Australian Indigenous communities, with the prevalence of gonorrhoea and chlamydia at more than $10 \%$ among the 16-19 age groups within some remote communities (Guy, Garton et al. 2011), and the diagnosis rates of gonorrhoea and chlamydia (673 and 1343 per 100000 population respectively) 30 and 3.5 times greater than those of nonIndigenous urban populations (at 22 and 378 per 100000 population respectively) (The Kirby Institute 2012). This is despite intensive STI interventions such as community screening of high coverage (Guy, Ward et al. 2012) over the last decade. The persistence of infection at high endemic levels in remote communities has led to questions about the validity of current approaches for control and prevention (Bowden and Fethers 2008).

In our previous modelling study (Hui, Gray et al. 2013), we investigated the roles of short-term population movement (of less than 6 months) on the persistence of infection in multiple small communities. We found that if some individuals seek additional sexual partners while they are away from home, then short term travel can contribute to STI persistence. Secondly, assuming the number of individuals away from home at a given time is held constant, then periodicity in mobility, due to changes in road accessibility between wet and dry seasons and the timing of annual cultural events for example, can result in STI prevalence being sustained at higher levels.

High population mobility is known to cause discontinuities in general health service delivery, leading to significant resource wastage and poor health outcomes (Prout 2008). The impact of discontinuity due mobility is likely to be significant on STI control strategies, given most of these controls focus on the younger, 16-25 age groups, who are often the most mobile (Biddle and Prout 2009). Furthermore, due to the small population size and vast physical distances between some of these communities, it is often impractical or even impossible to provide the same level of service for every location. Because of these limitations, it is of interest to identify strategies that can provide the largest reductions in STI prevalence given a fixed level of resources available.

In this study, we have extended our previous analysis and focused our investigation on the impact of short term periodic mobility on STI screening strategies. We also investigate the possible impacts on STI prevalence if, instead of a population-wide screening strategy of uniform coverage, location- and timespecific screening strategies are introduced instead.

\section{METHODS}

We developed an individual-based mathematical model of STI transmission to describe a sexually active population aged from 15 to 45 years. The model tracked age, gender, home and current location, sexual behaviour, and infection status on a daily basis for a period of 60 years (after initial model burn-in of 30 years). Individuals aged beyond 45 during simulation runs were to be removed from the population, but they will still have sex with their existing partners until the partnerships expired. For every individual removed from the model due to aging, a new individuals of age 15 (with no infections) will enter the population at their home location. These new individuals will have the same gender, home location and sexual behaviour (in terms of partner seeking frequency and likelihood of forming casual partnerships) as the removed individual. The model was extended to include analyses on the distribution of infection across multiple locations, as well as the impact of non-uniform (location- and time-specific) STI screening interventions on prevalence. A summary of the modelling methodology and structure follows, with the full implementation details described elsewhere (Hui, Gray et al. 2013).

The statistics for 100 simulation runs were generated for each intervention investigated. All events relating to an individual's sexual behaviour and movement were determined through a random number generator of fixed seed, and were the same across each intervention. The only parameter varied was the annual screening and treatment coverage. These will lead to changes in the number of infections and transmission in the population, as well as the overall duration of infection within the populations.

\subsection{Population demographics and movement}

The modelled population consists of 5000 individuals, corresponding approximately to the number of 15 to 45 years old in regions such as Alice Springs or Tennant Creek in the Northern Territory of Australia (Australian Bureau of Statistics 2009). The population is assumed to consist of one regional centre and four smaller remote satellite communities. Formation of partnerships and sexual contact between partners can occur if they are in the same location at the same time. Existing partnerships are maintained even if the partners move apart, and they can resume once they share the same location again. 
Hui et al., The role of short term population movement in sustaining STI prevalence in remote Australian Indigenous communities

We define an individual's usual place of residence as their "home location", and individuals currently located at their home location as residents. Individuals in this model cannot change their home locations, but are able to move to any other location temporarily before returning home. The regional centre is the home location of 4000 individuals, with each of the four remote communities being the home location of 250 individuals.

Individual mobility is implemented such that the proportion of the total population temporarily away from home at a given time is the same as reported in the 2006 census data (Biddle and Prout 2009). In our model, this ranges from $7.9 \%$ to $11.1 \%$ depending on age and gender (Hui, Gray et al. 2013). The candidates for movement are selected through a random pick process. As a simplified example, let us assume 100 individuals can be away from home at any time, and at a particular time step, 95 individuals are away from home. This means 5 residents will be randomly selected and move away from home in the next time step, (assuming none of the 95 non-residents have returned home since then). An individual's destination of movement is also determined via a random pick process, weighted by the population size at each possible destination. We based this assumption on the findings from studies of Australian remote Indigenous communities, which suggest that mobility is more closely associated with the availability of services, kinship or family ties (which are assumed to be more plentiful in larger populations) than with proximity (Biddle and Prout 2009).

The model uses three parameters to describe individual mobility as well as possible changes to individuals' behaviour as they move away from home. For our current analysis we also introduce a periodic variation denoted by the notation $(175 \%, 25 \%)$. This corresponds to an increase in the proportion of the population that is away to 1.75 times the yearly average during the first half of a year, and a reduction in the proportion to 0.25 times the yearly average during the second half of the year. Descriptions of these parameters, as well as the values used for all results generated in this study, are given in Table 1 below.

Table 1. Model parameters

\begin{tabular}{|l|l|}
\hline Description & Value \\
\hline $\begin{array}{l}\text { Probability (per day) of an individual with an existing partnership in their home } \\
\text { location seeking a new partner while away from home }\end{array}$ & 0.4 \\
\hline Proportion of the population that contributes to movement & $100 \%$ \\
\hline Number of days, on average, an individual stays away from home & 14 to 21 days \\
\hline Variation in the proportion of the population away from home over a 6-month period & $175 \%, 25 \%$ \\
\hline
\end{tabular}

\subsection{Properties of gonorrhoea and chlamydia}

The acquisition and natural history of infection for gonorrhoea and chlamydia follow identical pathways: susceptible, exposed, infected, recovered (not susceptible), and susceptible to reinfection. The natural histories of the two STIs are distinguished by the appropriate choice of parameter values, with the most important difference being the probability of infection being asymptomatic, which is much higher for chlamydia than for gonorrhoea. This means chlamydia infection usually lasts longer as most infections can only be detected and treated through screening. The complete list of parameter values used for both types of infection is described elsewhere (Hui, Gray et al. 2013).

\subsection{STI screening intervention}

In a recent review of STI interventions in Australia, it was found at least $44 \%$ of the population were screened and treated annually in many remote communities (Guy, Ward et al. 2012). However, the denominators associated with coverage were interpreted differently across communities. There was anecdotal evidence suggesting that screening coverage in some clinics often appeared to exceed $100 \%$ due to a mismatch between the number of residents (often used as the denominator) and the number of individuals the clinic serviced (refers as the "service population"). In addition, screening coverage alone does not provide a fair comparison with respect to the resources required as communities vary considerably in size and spread. Therefore, in this study we also consider the way screening coverage can be interpreted. We investigate the consequences of two interpretations: screening coverage is restricted by the number of residents that can be reached, or restricted by the resources available. For example, assume local screening coverage is $44 \%$. Under the first interpretation, this means that only $44 \%$ of the local population can access screening (e.g. unaware or unwilling to anticipate screening), even if more resources (e.g., staff) are shifted from other locations. In the second interpretation, $44 \%$ of the local population are accessible for screening currently, but 
Hui et al., The role of short term population movement in sustaining STI prevalence in remote Australian Indigenous communities

more residents can be reached if resources can be shifted to that location at the expense of others. For simplicity we assume screening is $100 \%$ sensitive and specific, treatment will be provided immediately upon detection of infection, and treatment is $100 \%$ effective.

We assume when and where an individual moves are unknown to health care service providers, and screening has to be organised and scheduled ahead based on existing local residency data. As individual cannot change their residency (i.e. their home locations) in the model, therefore the denominator defining screening coverage will always be fixed at 4000 for the regional centre (denoted as L0 in the results), and 250 for the remaining four remote communities (L1-L4).

\section{RESULTS}

\subsection{The role of mobility in sustaining STI prevalence}

Our previous analysis found gonorrhoea and chlamydia could persist in multiple small locations if mobile individuals who are already in a regular partnership are allowed to seek additional partners while away from home. Furthermore, if individuals only stayed away from home for a short period, and there was periodicity in mobility, then STIs were more likely to persist at higher level of prevalence (Hui, Gray et al. 2013).

In our current analysis, we are interested in the spread of infection under various screening interventions. Using the parameter values defined in Table 1, the model is able to sustain a gonorrhoea and chlamydia prevalence of $8.0 \%\left(25^{\text {th }}-75^{\text {th }}\right.$ percentile: $7.4 \%$ and $\left.8.8 \%\right)$ and $9.4 \%\left(25^{\text {th }}-75^{\text {th }}\right.$ percentile: $8.5 \%$ and $\left.10.5 \%\right)$, respectively, without any screening intervention, and $7.5 \%$ and $6.5 \%$, respectively, under annual screening of $44 \%$ coverage for all locations.

\subsection{Distribution of infection across multiple locations}

The result in Figure 1 shows the distributions of population and STI prevalence across the five locations during the last year of simulation runs, where the distributions and STI prevalence would have remained stable for at least 35 years. It shows, on average, $72 \%$ of the population are to be found at location L0. However, the infections are not spread uniformly across the locations, with more than $80 \%$ of the infected found in L0 for both infections.

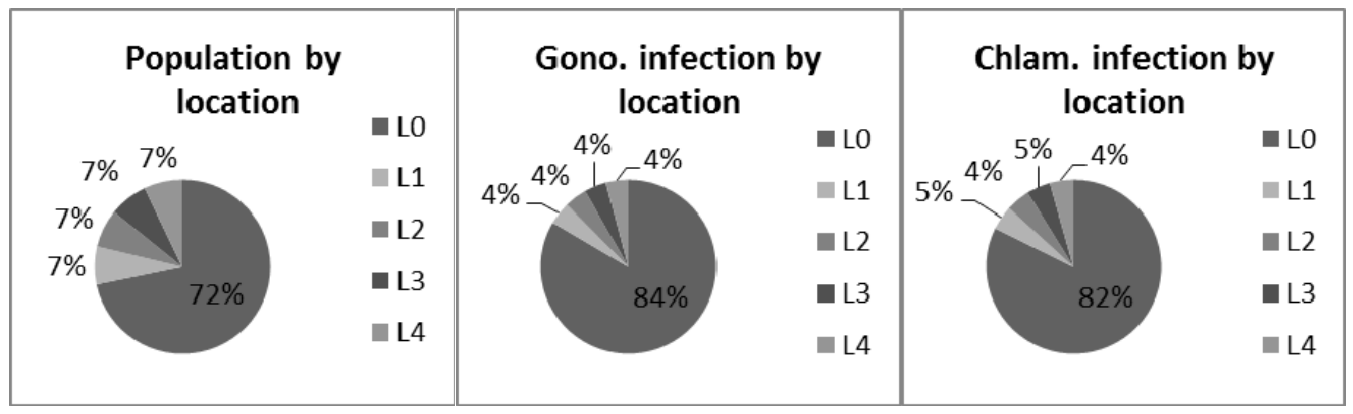

Figure 1. Distribution of population and STI prevalence across all locations over last 35 years of simulaion runs (averaged over simulation 100 runs).

\subsection{Location-specific screening interventions}

The result in Figure 1 shows STI prevalence is not evenly distributed across locations, suggesting that an alternate approach is could be to implement different levels of screening coverage in different locations. In this section, we investigate the impact of such an approach by introducing location-specific screening interventions to our model.

Location-specific screening in this model follows a pre-defined schedule, which is generated by randomly selecting a fixed number of residents at the start of each year. Screenings are distributed evenly across the scheduled period. For example, a screening coverage of 44\% per 360 days in L0 means 1760 (out of 4000) residents are scheduled to be screened. This translates to 40 days during which four residents were scheduled to be screened per day, and 320 days during which five residents were scheduled to be screened per day. We assumed individuals can only be screened if they were at their home locations during their scheduled screening day, and individuals would not alter their mobility pattern due to screening interventions.

We examined a range of location-specific screening interventions, and they are classified as follows. In 'All locations', 44\% of the residents in each location are scheduled to be screened once per year, with 1760 individuals scheduled for screening in L0, and 110 in each of L1 to L4. In 'Regional centre only', 44\% of the 
Hui et al., The role of short term population movement in sustaining STI prevalence in remote Australian Indigenous communities

residents in L0 are scheduled to be screened once per year, with no screening for residents at other locations. Note that even though 1760 residents of L0 are scheduled to be screened for this intervention, they are not necessarily the same 1760 residents scheduled to be screened in 'All locations' intervention. In 'Regional centre only +', 2200 (so 44\% of total population of 5000) individuals are scheduled for screening in one year, all of whom are residents of L0. In 'Remote only', 44\% of the residents in L1-L4 are scheduled to be screened once per year, with no screening for L0. In 'Remote only +', again 2200 individuals are scheduled for screening, with 550 individuals scheduled for screening in each of L1 to L4, and no screening of residents at L0. In the last scenario, the number of screenings is larger than the number of local residents, so every resident in L1 to L4 is scheduled to be screened at least twice per 360 days, with 50 of them scheduled to be screened three times per 360 days.

If we assume screening coverage is only limited by the resources available to the whole population (or that resources can be shifted between locations such that the total number of scheduled screenings will always be 2200 ), then the greatest reduction of prevalence at the end of simulation runs occurs at 'Regional centre only +'. However, this assumes localised screening coverage in the regional centre could increase from $44 \%$ to $55 \%$ per year. If screening coverage is fixed at $44 \%$ of local residents (i.e. interventions without ' + '), then an intervention applied to all locations would lead to the lowest prevalence, with the exception gonorrhoea, where annual screening of $44 \%$ coverage in remote locations (either by itself or part of 'All locations' intervention strategy) makes no significant change to the median population prevalence, with the absolute differences to prevalence of $0.02 \%$, or one individual, across 100 simulation runs. In addition, the differences in median prevalence between uniform and location-specific interventions are only significant for chlamydia (at 5\% significance level), with 'Regional centre only + ' the only strategy that performs better than uniform screening for all locations.

Table 2. The impact of location-specific intervention. The medians which were significantly different (at the $5 \%$ significance level) to the medians under uniform screening for all locations are shown in bold.

\begin{tabular}{|c|c|c|c|c|c|}
\hline \multirow{2}{*}{ Intervention type } & \multirow{2}{*}{$\begin{array}{c}\text { Number of } \\
\text { screenings scheduled } \\
\text { in one year }\end{array}$} & \multicolumn{2}{|c|}{$\begin{array}{c}\text { Gonorrhoea prevalence at the end of } \\
60 \text { years }(\%)\end{array}$} & \multicolumn{2}{|c|}{$\begin{array}{c}\text { Chlamydia prevalence at the end of } \\
60 \text { years }(\%)\end{array}$} \\
\hline & & Median & $25^{\text {th }}-75^{\text {th }}$ percentile & Median & $25^{\text {th }}-75^{\text {th }}$ percentile \\
\hline All locations & 2200 & 7.54 & $6.61-8.20$ & 6.57 & $5.53-7.63$ \\
\hline Regional centre only & 1760 & 7.52 & $6.95-8.28$ & 7.21 & $6.56-7.84$ \\
\hline Regional centre only + & 2200 & 7.09 & $6.30-7.81$ & 5.95 & $5.22-7.00$ \\
\hline Remote only & 440 & 7.96 & $7.31-8.58$ & 9.05 & $8.15-9.77$ \\
\hline Remote only + & 2200 & 7.60 & $7.14-8.57$ & 8.26 & $7.50-9.14$ \\
\hline
\end{tabular}

\subsection{Location- and time-specific screening intervention}

Screening coverage could also vary in time in accordance with periodicity in mobility patterns. In our model, periodicity is defined in a half-yearly cycle, therefore one option is to organise screenings in half yearly cycles also.

In Table 3, the total number of screenings scheduled is the same as for the corresponding intervention types listed in Table 2, but the timing is organised in four different ways: 1) completely in-sync, where all screenings are scheduled to occur during the high mobility period; 2) completely out-of-sync, where all screenings are scheduled to occur during the low mobility period; 3) proportional in-sync, where $75 \%$ of screenings are scheduled to occur during the high mobility period, and; 4) proportional out-of-sync, where $75 \%$ of screenings are scheduled to occur during the low mobility period.

The results in Table 3 show that time-specific screening interventions can lead to lower STI prevalence than continuous interventions. However, apart from chlamydia screening interventions involving the regional centre, the differences in median prevalence are not statistically significant (at the $5 \%$ significance level).

The results suggest that out-of-sync interventions involving the regional centre perform better than their insync counterparts, as most residents would be at home during low mobility periods. For example, for screening interventions that involve all locations, up to $9 \%$ of the scheduled screenings are not carried out due to residents being away during their scheduled screening days. This percentage is increased to $12 \%$ if the screening schedule is completely in-sync with periodicity, and is reduced to $3 \%$ if screening schedule is completely out-of-sync. 
Hui et al., The role of short term population movement in sustaining STI prevalence in remote Australian Indigenous communities

Table 3. The impact of location and time-specific intervention. The medians that were significantly different (at the $5 \%$ significance level) to the medians in Table 2 are shown in bold. The medians that were lower and significantly different (at 5\% significance level) to the medians under continuous screening for all locations are highlighted in grey.

\begin{tabular}{|c|c|c|c|c|c|c|}
\hline \multirow{2}{*}{ Intervention type } & \multicolumn{2}{|c|}{ Number of screenings scheduled } & \multicolumn{2}{|c|}{$\begin{array}{l}\text { Gonorrhoea prevalence at the } \\
\text { end of } 60 \text { years }(\%)\end{array}$} & \multicolumn{2}{|c|}{$\begin{array}{c}\text { Chlamydia prevalence at the end } \\
\text { of } 60 \text { years }(\%)\end{array}$} \\
\hline & $\begin{array}{l}\text { During high } \\
\text { mobility period }\end{array}$ & $\begin{array}{l}\text { During low } \\
\text { mobility period }\end{array}$ & Median & $\begin{array}{l}25^{\text {th }}-75^{\text {th }} \\
\text { percentile }\end{array}$ & Median & $\begin{array}{l}25^{\text {th }}-75^{\text {th }} \\
\text { percentile }\end{array}$ \\
\hline \multirow{4}{*}{ All locations } & 2000 & 0 & 7.48 & $6.54-8.29$ & 6.31 & $5.71-7.51$ \\
\hline & 0 & 2000 & 7.25 & $6.35-7.97$ & 5.30 & $4.67-6.15$ \\
\hline & 1652 & 548 & 7.54 & $6.66-8.15$ & 6.46 & $5.83-7.36$ \\
\hline & 548 & 1652 & 7.39 & $6.64-8.03$ & 6.63 & $5.87-7.39$ \\
\hline \multirow{4}{*}{ Regional centre only } & 1760 & 0 & 7.47 & $6.66-8.19$ & 6.75 & $5.96-7.58$ \\
\hline & 0 & 1760 & 7.27 & $6.45-7.92$ & 5.55 & $4.65-6.23$ \\
\hline & 1320 & 440 & 7.25 & $6.74-8.28$ & 6.54 & $5.85-7.36$ \\
\hline & 440 & 1320 & 7.41 & $6.66-8.33$ & 6.95 & $6.11-7.60$ \\
\hline \multirow{4}{*}{ Regional centre only +} & 2200 & 0 & 7.26 & $6.49-8.05$ & 5.33 & $4.49-6.26$ \\
\hline & 0 & 2200 & 6.89 & $5.99-7.56$ & 4.56 & $3.84-5.59$ \\
\hline & 1650 & 550 & 7.04 & $6.38-7.99$ & 5.55 & $4.70-6.55$ \\
\hline & 550 & 1650 & 7.19 & $6.46-8.03$ & 5.62 & $5.04-6.62$ \\
\hline \multirow{4}{*}{ Remote only } & 440 & 0 & 8.00 & $7.42-8.64$ & 9.29 & $8.33-9.98$ \\
\hline & 0 & 440 & 8.01 & $7.52-8.66$ & 9.18 & $8.46-9.93$ \\
\hline & 332 & 108 & 8.19 & $7.34-8.91$ & 9.21 & $8.55-9.83$ \\
\hline & 108 & 332 & 8.16 & $7.36-8.78$ & 9.31 & $8.49-10.17$ \\
\hline \multirow{4}{*}{ Remote only +} & 2200 & 0 & 7.86 & $7.10-8.57$ & 8.74 & $7.82-9.58$ \\
\hline & 0 & 2200 & 7.87 & $7.15-8.48$ & 8.44 & $7.79-9.51$ \\
\hline & 1648 & 552 & 8.07 & $7.22-8.82$ & 8.62 & $7.98-9.26$ \\
\hline & 552 & 1648 & 7.87 & $7.31-8.74$ & 8.52 & $7.79-9.24$ \\
\hline
\end{tabular}

\section{DISCUSSION AND CONCLUSIONS}

Our modelling result show that STIs are not distributed evenly in a mobile population, with infected individuals more likely to be found in populous locations. It is reasonable to assume that interventions would be most effective if they are focused on the most prevalent, and in terms of this model, the most populous, location and our findings support this hypothesis. More poignantly, the results suggest that annual screening coverage of $44 \%$ in remote locations is not sufficient to reduce prevalence, with median prevalence for all 'Remote only' interventions (i.e., those that do not include the regional centre) not significantly different to the median under no screening interventions (at the 5\% significance level). If screening is available for remote locations only, then the reduction in STI prevalence is minimal unless there is a sizeable increase in screening coverage and/or frequency.

If the resources available for screening are limited but can be relocated, then allocating all screenings to the regional centre leads to the large reduction in prevalence. However this finding assumes screening would be equally effective in any location. This might not hold true in reality as, for example, it might be easier to locate a specific high risk individual or trace the spread of infection in small populations, while screening of high coverage might be difficult to maintain in larger populations.

The results for time-specific intervention strategies suggest that screening should be scheduled during periods of low mobility. There are two reasons for this: 1) a time delay between mobility cycle and its impact on STI prevalence, and 2) residents are more likely to be at home and available for their scheduled screening during low mobility periods. Screening interventions should be designed such that they reach the maximum number of individuals and, for this model, this occurs when most individuals are at home. However, it should be noted that this finding is only valid for interventions based on a pre-defined schedule. For opportunistic 
Hui et al., The role of short term population movement in sustaining STI prevalence in remote Australian Indigenous communities

interventions, additional resources should be allocated during periods of high population mobility due to the possible increase to population a local clinic needs to be serviced.

In this study a number of simplifying assumptions were made. It was assumed that individuals are more likely drawn to larger populations due to availability of facilities and services. We also assumed travellers only stayed in one location per episode of travel before returning home and did not consider circular mobility (Taylor 2009). Both of these assumptions were made due to the lack of sufficiently detailed data on temporary mobility. Alternative approaches, such as the use of transits networks or random graphs could be a better option if detailed travel data become available.

Another important assumption made is the level of sexual activity, especially among non-residents. While sexual activity for residents in this model is calibrated to available data (Bryant, Ward et al. 2011), it is unclear if these data are applicable to non-residents. In this study, we made the broad assumption that nonresidents would not seek partners more or less frequently than residents, but some non-residents who are already in partnerships will 'identify' themselves as single, and seek new partners at the same rate as when they are single. This can be considered a compromise between two extremes 1) where individuals will not engage in any sexual activity while they are away from their partners (in this case mobility will have reduce STI transmission); and 2) where individuals will increase their levels of sexual activity when they are away from their partners (in this case mobility will increase transmission). In reality, the sexual activity of mobile individuals will lie between these extremes, and is likely to be specific to individual or population-specific factors such as gender, age, and location.

This study highlights the role that temporary mobility may have in sustaining the high levels of STI prevalence observed in remote Indigenous populations of Australia. STIs are more likely to be found in the populous locations, and screening that included targets regional centres is scheduled for periods of low mobility are predicted to be the most effective in reducing prevalence.

\section{ACKNOWLEDGMENTS}

This study was funded by a National Health and Medical Research Council Program Grant (568971). The Kirby Institute is funded by the Australian Government Department of Health and Ageing and is affiliated with the Faculty of Medicine, University of New South Wales. The views expressed in this publication do not necessarily represent the position of the Australian Government.

\section{REFERENCES}

Australian Bureau of Statistics (2009). Experimental Estimates and Projections, Aboriginal and Torres Strait Islander Australians, 1991 to 2006 (cat. no. 3238.0). Canberra.

Biddle, N. and S. Prout (2009). "The geography and demography of Indigenous temporary mobility: an analysis of the 2006 census snapshot." Journal of Population Research 26: 305-326.

Bowden, F. J. and K. Fethers (2008). "“Let's not talk about sex": reconsidering the public health approach to sexually transmissible infections in remote Indigenous populations in Australia." Medical Journal of Australia 188(3): 182-184.

Bryant, J., J. Ward, H. Worth, P. Hull, S. Solar and S. Bailey (2011). "Safer sex and condom use: a convenience sample of Aboriginal young people in New South Wales." Sexual Health 8: 378-383.

Guy, R., L. Garton, Taylor-Thompson D, B. Silver, B. Hengel, J. Knox, S. McGregor, A. Rumbold, J. Ward and J. Kaldor (2011). The 2010 baseline prevalence study conducted by the STRIVE trial. Australasian Sexual Health Conference. National Convention Centre, Canberra, ACT, Australia.

Guy, R., J. S. Ward, K. S. Smith, J. Y. Su, R. L. Huang, A. Tangey, S. Skov, A. Rumbold, B. Silver, B. Donovan and J. M. Kaldor (2012). "The impact of sexually transmissible infection programs in remote Aboriginal communities in Australia: a systematic review." Sex Health 9(3): 205-212.

Hui, B. B., R. T. Gray, D. P. Wilson, J. S. Ward, A. M. A. Smith, D. J. Philp, M. G. Law, J. S. Hocking and D. G. Regan (2013). "Population movement can sustain STI prevalence in remote Australian indigenous communities." BMC Infectious Diseases 13: 188.

Prout, S. (2008). The entangled relationship between Indigenous spatiality and government service delivery. CAEPR Working Paper No. 41. Canberra, Centre for Aboriginal Economic Policy Research, ANU.

Taylor, J. (2009). "Indigenous demography and public policy in Australia: population or peoples?" Journal of Population Research 26: 115-130.

The Kirby Institute (2012). Bloodborne viral and sexually transmitted infections in Aboriginal and Torres Strait Islander People: Surveillance and Evaluation Report 2012. Sydney, NSW, The Kirby Institute, the University of New South Wales. 